Check for updates

Cite this: RSC Adv., 2018, 8, 32283

Received 25th April 2018 Accepted 28th August 2018

DOI: $10.1039 / \mathrm{c} 8 \mathrm{ra03575j}$

rsc.li/rsc-advances

\section{Acidolysis-dominated pretreatment elevates distillation yield and impacts composition, antioxidant and antifungal activities of essential oil from Cuminum cyminum seeds}

\author{
Zhong Zhang, (D) ab Qiang Qin, ${ }^{b}$ Ruojun Ding, ${ }^{b}$ Yibing Xia, ${ }^{b}$ Libo Xiong, ${ }^{b}$ Yang Bi ${ }^{\star a b}$ \\ and Dov Prusky
}

Proper pretreatment of herbal material containing essential oils (EOs) could enhance its volatile components release through either removing physical barriers or conquering chemical bonds and thereby improve hydrodistillation yield. In this regard, a trial pretreatment including pulverization, enzymolysis, short time microwave irradiation and acidolysis of Cuminum cyminum seeds was integrated into the essential oil (EO) preparation to elevate the EO yield. On the basis of Plackett-Burman design analysis, three parameters (acidolysis duration, $\mathrm{HCl}$ concentration of acidolysis and sieving mesh) were significant for the EO preparation. Box-Behnken design based optimization of the remaining factors concluded that the optimal pretreatment was pulverizing the seeds to 40 mesh and implementing 45 min acidolysis in $2.5 \mathrm{M} \mathrm{L}^{-1} \mathrm{HCl}$ wherein the predicted $\mathrm{EO}$ yield of $3.78 \%$ was close to that of the experimental value $3.86 \%$. This pretreatment produced an EO yield increase of $50.78 \%$ over the control sample of raw seeds (2.56\%). In total 53 components were identified in the acidolysis-pretreated cumin EO (AEO) whilst 47 components were identified in the control cumin EO (CEO). In both AEO and CEO, cuminaldehyde was the predominant common component, but the AEO contained more phenols $(0.51 \%$ vs. $0.18 \%)$ and alcohols $(7.76 \%$ vs. $0.18 \%)$ than the CEO did. The compositional features gave the AEO mightier antioxidant potency and stronger antifungal efficacy against four postharvest fungi, viz. Alternaria alternata, Penicillium expansum, Trichothecium roseum and Fusarium sulphureum, as compared with the situations of CEO. In conclusion, the pretreatment elevates the hydrodistillation yield, modifies the EO chemical profiles and confers stronger antioxidant and antifungal activities upon cumin EO.

\section{Introduction}

Cumin (Cuminum cyminum) seed is the second most consumed spice after black pepper in the world and is mainly cultivated in Arabia, India, China and some countries adjoining the Mediterranean Sea. ${ }^{\mathbf{1}}$ Traditionally the seed has been extensively used for various medicinal, culinary, and aromatic purposes, ${ }^{2}$ but to date more new bioactivities of its essential oil (EO) have been investigated for deleterious microorganism inhibition, ${ }^{\mathbf{1}, 3}$ grainstorage, ${ }^{4}$ and phytophagous pest control ${ }^{5}$ objectives. Moreover, cumin EO exerts anti-inflammatory effects in LPS-stimulated RAW264.7 cells. ${ }^{6}$ This effect is of great importance since inflammation is regarded as a cause of chronic disease ${ }^{7}$ which accounts for nearly two-thirds of deaths worldwide, and the

${ }^{a}$ College of Horticulture, Gansu Agricultural University, Lanzhou 730070, China. E-mail: beyang62@163.com; foodgau@126.com

${ }^{b}$ College of Food Science and Engineering, Gansu Agricultural University, Lanzhou 730070, China emergence of chronic diseases as the predominant challenge to global health is undisputed. ${ }^{8}$

As a value-added product of cumin seeds processing, the EO has a disappointing low yield, and elevating this yield is of significance for increasing the profitability of the EO production. Hydrodistillation, the typical EO extraction approach can obtain the highest yield, but the yield is still associated with the intrinsic nature of the raw materials. ${ }^{9}$ Modifications of this nature seem an accessible vehicle for fully tapping the latent EO components in the material, thereby elevating the yield within a short space of time. EO compounds are found in intracellular spaces, more than on the surface of the vegetal cell. ${ }^{10}$ For dry herbal materials like cumin seeds, pulverization can crumble the tissues of the materials and partly remove any physical barrier to EO releasing. ${ }^{11}$ But normal grinding can raise the product temperature to $42-95{ }^{\circ} \mathrm{C}$ and cause loss of volatile components up to $40 \%,{ }^{12}$ thus the optimal extent of pulverization deserves investigation. Furthermore, EOs were accumulated in the secretory structures of plant tissues and cellulolytic degradation of such structures can facilitate releasing and 
diffusion of EO components. ${ }^{\mathbf{1 3}}$ More importantly, particular amount of EO components are glycosidically bound ${ }^{\mathbf{1 4}}$ and thereupon are generally hydrophilic, nonvolatile, and unobtainable with simple hydrodistillation. Hydrolytically breaking of such glycosidical bonds can liberate aglycone moieties that could be latent EO components, whereas the effect of acidolysis on cumin EO lacks reports. External microwave assistance with ionic water hydrodistillation shortens the extraction time without obvious influence on EO constituents, ${ }^{15}$ but this simultaneous application of microwave irradiation require more complex facilities. Interestingly, microwave-heated cumin seeds gain higher EO yield than conventionally roasted samples do in then hydrodistillation, ${ }^{\mathbf{1 6}}$ but the effect of microwave irradiation, prior to distillation, on cumin EO yield was not explored.

In documented investigations into the effect of pretreatment on cumin EO preparation, the most researches focused on single factor introduced, whilst the application of hyphenated compound factors in the treatment of cumin seeds is less analyzed. Generally, these introduced treatments might have some influences on the compositional and bioactive attributes of the EO, thus evaluation of these influences is necessary when considering the feasibility of the application of these potential treatments in production. As stereotypical bioactivities of the EO, antioxidant and antimicrobial activities were assessed as basic aspects when the bioactive quality of different EOs was compared. ${ }^{17}$

In the present work, the Plackett-Burman design (PBD) and Box-Behnken design (BBD) based analyses were employed for the determination of key factors and their best function zones where an optimal EO yield could be procured. To evaluate the influence of the introduced treatments on the chemical attributes and bioactivities of the EO, the composition, in vitro antioxidant potency and antifungal activity of the EO were analyzed. To the best of our knowledge, this investigation was the first one that integrates acidolysis into the extraction of EOs with the intention of liberating the bound EO components.

\section{Material and methods}

\subsection{Cumin seeds}

Cumin (C. cyminum cv. Dunyu no. 1) seeds newly harvested were obtained from the grower Dunhuang Seed Co., Ltd. (Dunhuang, China).

\subsection{Chemicals and reagents}

Cellulase from Trichodermavirid (Solarbio, Japan) was used for treatment of cumin seed slurry. Chromatographic level $n$ hexane for sample dilution and homologous series of $\mathrm{C}_{5}-\mathrm{C}_{24} n^{n}$ alkanes for gas chromatography-mass spectrometry (GC-MS) analysis were purchased from Sigma-Aldrich (Steineheim, Germany). The 2,2-diphenyl-1-picrylhydrazyl(DPPH),2,2'azino-bis(3-ethylbenzothiazoline-6-sulfonic acid)(ABTS), (2,4,6-trislz-pyridyl)-s-triazine(TPTZ), $\beta$-carotene and ( \pm -6hydroxy-2,5,7,8-tetramethylchromane-2-carboxylic acid (Trolox) were purchased from Sigma-Aldrich (St. Louis, MO, USA).
All other chemicals were analytical grade and purchased from the Sinopharm Chemical Reagent (Shanghai, China).

\subsection{Essential oil preparation}

2.3.1. Pretreatment of cumin seeds. The cumin seeds were pulverized into powders passing through standard sieves with experimental mesh size. Samples of $100 \mathrm{~g}$ powders were subsequently subjected to enzymolysis as slurry with $\mathrm{pH}$ proper to cellulase. Then the slurry was irradiated $(800 \mathrm{~W})$ using a microwave oven (WD800ASL23, Galanz, China) in an alternating pattern of $10 \mathrm{~s}$ irradiation and $20 \mathrm{~s}$ off-interval to avoid releasing of volatiles before distillation. Adjust the ratio of sample to liquid and the $\mathrm{HCl}$ intensity and then conduct acidolysis with water incubation at set temperature and for certain duration. Process parameters viz. sample particle size (controlled with sieving mesh sizes), cellulase concentration, enzymolytic temperature, $\mathrm{pH}$ and duration, microwave irradiation duration accumulated, acid concentration and duration of acidolysis, all were adjusted as per the experimental design (Table 1) and trialed. Cumin seeds without pulverizing, cellulase, acid or microwave treatments were used as control.

2.3.2. EO hydrodistillation. The cumin slurry was hydrodistilled for $3 \mathrm{~h}$ via a Clevenger type apparatus according to the Pharmacopoeia Committee of People's Republic China. ${ }^{\mathbf{1 8}}$ Considering the residual $\mathrm{HCl}$ in the EOs from treated samples, when finished $3 \mathrm{~h}$ distillation, replace the distilled cumin slurry with sodium hydroxide solution of $\mathrm{pH}$ 8.75-9.0 and continue a faux distillation until the EOs have a similar $\mathrm{pH}$ with control EO ( $\mathrm{pH}$ 3.86). The EOs were then collected and dried over anhydrous sodium sulfate and stored in sealed amber vials and kept at $4{ }^{\circ} \mathrm{C}$ prior to analysis. The EO yields (w/w) were calculated on a dry weight basis of cumin powder.

2.3.3. Experimental design. To determine what implications the previous treatments have on the EO yield, the

Table 1 Plackett-Burman design for checking the trialed extractions of essential oil using different levels of factors ${ }^{a}$

\begin{tabular}{|c|c|c|c|c|c|c|c|c|c|c|}
\hline \multirow[b]{2}{*}{ Run } & \multicolumn{9}{|c|}{ Factors } & \multirow[b]{2}{*}{ Yield (\%) } \\
\hline & $X_{1}$ & $X_{2}$ & $X_{3}$ & $X_{4}$ & $X_{5}$ & $X_{6}$ & $X_{7}$ & $X_{8}$ & $X_{9}$ & \\
\hline 1 & 20 & 1 & 4.5 & 47 & 30 & 60 & 3 & 75 & $1 / 6$ & $2.80 \pm 0.02$ \\
\hline 2 & 70 & 1 & 5.5 & 47 & 30 & 30 & 3 & 45 & $1 / 6$ & $2.53 \pm 0.01$ \\
\hline 3 & 70 & 3 & 5.5 & 47 & 30 & 60 & 1 & 45 & $1 / 6$ & $2.29 \pm 0.05$ \\
\hline 4 & 70 & 1 & 5.5 & 47 & 60 & 60 & 3 & 45 & $1 / 6$ & $2.42 \pm 0.08$ \\
\hline 5 & 20 & 1 & 5.5 & 43 & 30 & 60 & 3 & 45 & $1 / 8$ & $2.49 \pm 0.02$ \\
\hline 6 & 20 & 1 & 4.5 & 43 & 30 & 30 & 1 & 45 & $1 / 8$ & $2.38 \pm 0.06$ \\
\hline 7 & 20 & 1 & 5.5 & 47 & 30 & 30 & 3 & 45 & $1 / 8$ & $2.57 \pm 0.01$ \\
\hline 8 & 70 & 1 & 5.5 & 47 & 60 & 30 & 1 & 75 & $1 / 8$ & $2.48 \pm 0.01$ \\
\hline 9 & 70 & 1 & 4.5 & 43 & 60 & 60 & 3 & 75 & $1 / 8$ & $2.46 \pm 0.01$ \\
\hline 10 & 70 & 3 & 5.5 & 43 & 60 & 60 & 1 & 75 & $1 / 8$ & $2.45 \pm 0.02$ \\
\hline 11 & 70 & 3 & 4.5 & 43 & 30 & 30 & 3 & 45 & $1 / 6$ & $2.56 \pm 0.02$ \\
\hline 12 & 20 & 3 & 4.5 & 43 & 60 & 30 & 1 & 45 & $1 / 6$ & $2.49 \pm 0.04$ \\
\hline
\end{tabular}

${ }^{a} X_{1}=$ sieving mesh, $X_{2}=$ enzyme concentration $\left(10^{4} \mathrm{U} \mathrm{L}^{-1}\right), X_{3}=$ enzymolysis $\mathrm{pH}, X_{4}=$ enzymolysis temperature $\left({ }^{\circ} \mathrm{C}\right), X_{5}=$ enzymolysis duration $(\mathrm{min}), X_{6}=$ microwave irradiation duration (s), $X_{7}=\mathrm{HCl}$ concentration of acidolysis $\left(\mathrm{M} \mathrm{L}^{-1}\right), X_{8}=$ acidolysis duration (min), $X_{9}$ $=$ sample to liquid ratio. For each factor, the small values represent its low level $(-1)$ and the large values represent its high level $(+1)$. 
approach concerning single factor at a time for its level selection is neither efficient nor capable of finding any interaction between these factors, but first and second order polynomial design allied can solve the problem well. PBD employs the first order polynomial to screen out significant factors and their further optimization can be fulfilled by BBD based response surface methodology. ${ }^{19}$

2.3.3.1. Single factor screening. For screening the factors that significantly $(P<0.05)$ influence the EO yield, candidate factors (sieving mesh, cellulase concentration, temperature, $\mathrm{pH}$ and duration for enzymolysis, duration of microwave irradiation, acidolysis $\mathrm{HCl}$ concentration and duration, and ratio of cumin powder to liquid) were examined with the PBD analysis. Each variable was tested at low $(-1)$ and high $(+1)$ levels and twelve trials were performed without consideration of any interactive effects and every trial was conducted in triplicate. The values of these levels were represented in Table 1 . The first order polynomial equation for PBD was as following:

$$
Y=A_{0}+\sum_{i=1} A_{i} X_{i}
$$

where $Y$ is the EO yield response, $A_{0}$ is intercept, $A_{i}$ is the linear regression coefficient for $i^{\text {th }}$ factor, $X_{i}$ is the level of the independent factor. The analysis of variance (ANOVA) was used to determine main effects of parameters. The normalized results of the experimental design were evaluated at a $5 \%$ significant level and illustrated by standardized Pareto chart with a vertical line as an indicator beyond which extending bars represent the factors that are statistically significant and were considered for further experiments.

2.3.3.2. Full factorial design. Each remained factor was coded (Table 2) at three levels, i.e. low $(-1)$, medium (0) and high $(+1)$ and a total of 17 experimental runs (Table 3) were conducted.

Fit the BBD experimental data into second order polynomial equation, via regression analysis, as following:

$$
Y=A_{0}+\sum_{i=1}^{k} A_{i} X_{i}+\sum_{i=1}^{k} A_{i i} X_{i}^{2}+\sum_{i=1}^{k} \sum_{j=i+1}^{k-1} A_{i j} X_{i} X_{j}
$$

where $Y$ is the EO yield response, $X$ is a variable, and $A$ is a regression coefficient. ANOVA was carried out to determine $F$ value for the significant $(P<0.05)$ linear, quadratic and interaction effect. Lack of fitness was also determined for the response model and fitness of polynomial equation was estimated using coefficient of determination $\left(R^{2}\right)$. Contour plots illustrating the impact of the remained factors on the EO yield were generated based on the established model.

Table 2 Coded levels of independent variables for Box-Behnken design

\begin{tabular}{llcl}
\hline Factor & Low & Medium & High \\
\hline$X_{1}$ mesh size & 20 & 40 & 70 \\
$X_{2}$ HCl concentration $\left(\mathrm{M} \mathrm{L}^{-1}\right)$ & 1.5 & 2 & 2.5 \\
$X_{3}$ acidolysis duration $(\mathrm{min})$ & 45 & 60 & 75
\end{tabular}

Table 3 Independent and dependent variables in Box-Behnken

\begin{tabular}{|c|c|c|c|c|c|}
\hline \multirow[b]{2}{*}{ Run } & \multirow[b]{2}{*}{$X_{1}$} & \multirow[b]{2}{*}{$X_{2}$} & \multirow[b]{2}{*}{$X_{3}$} & \multicolumn{2}{|l|}{ Yield (\%) } \\
\hline & & & & Experimental & Predicted \\
\hline 1 & -1 & 0 & 1 & $2.54 \pm 0.01$ & 2.51 \\
\hline 2 & -1 & -1 & 0 & $2.36 \pm 0.01$ & 2.30 \\
\hline 3 & 1 & -1 & 0 & $2.62 \pm 0.02$ & 2.53 \\
\hline 4 & -1 & 1 & 0 & $2.76 \pm 0.02$ & 2.85 \\
\hline 5 & 1 & 0 & 1 & $2.79 \pm 0.03$ & 2.80 \\
\hline 6 & 1 & 1 & 0 & $2.80 \pm 0.01$ & 2.85 \\
\hline 7 & 0 & 0 & 0 & $2.86 \pm 0.02$ & 2.95 \\
\hline 8 & 0 & -1 & 1 & $2.90 \pm 0.01$ & 2.99 \\
\hline 9 & 0 & 0 & 0 & $2.93 \pm 0.03$ & 2.95 \\
\hline 10 & 1 & 0 & -1 & $2.95 \pm 0.02$ & 2.98 \\
\hline 11 & 0 & 0 & 0 & $2.98 \pm 0.01$ & 2.95 \\
\hline 12 & 0 & 0 & 0 & $2.99 \pm 0.02$ & 2.95 \\
\hline 13 & 0 & 0 & 0 & $3.01 \pm 0.02$ & 2.95 \\
\hline 14 & 0 & -1 & -1 & $3.04 \pm 0.08$ & 3.10 \\
\hline 15 & -1 & 0 & -1 & $3.06 \pm 0.08$ & 3.05 \\
\hline 16 & 0 & 1 & 1 & $3.23 \pm 0.06$ & 3.17 \\
\hline 17 & 0 & 1 & -1 & $3.86 \pm 0.06$ & 3.78 \\
\hline
\end{tabular}
experiment design

\subsection{GC-MS analysis}

Chemical characteristic of EO was determined on a gas chromatograph model Trace 1310 with TG-WAX $(60 \mathrm{~m} \times 0.25 \mathrm{~mm}$, $0.25 \mu \mathrm{m}$ ) capillary column in tandem with ISQ single quadrupole mass detector (Thermo Scientific, USA). Helium was carrier

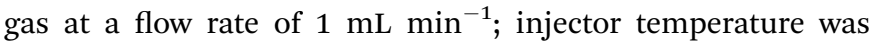
$250{ }^{\circ} \mathrm{C}$. Column temperature was programmed at $60{ }^{\circ} \mathrm{C}$ isothermal for $4 \mathrm{~min}$, and then increased to $240{ }^{\circ} \mathrm{C}$ at a rate of $4{ }^{\circ} \mathrm{C} \mathrm{min}^{-1}$ and held isothermal for $15 \mathrm{~min}$. The split ratio was $1: 20$, ionization voltage $70 \mathrm{eV}$; ion source temperature $200{ }^{\circ} \mathrm{C}$; mass scan range: 40-500 mass units; detector temperature $280{ }^{\circ} \mathrm{C}$. The injection volume was $1.0 \mu \mathrm{L}$ after $1 \%(\mathrm{v} / \mathrm{v})$ dilution of the EO with $n$-hexane. The percentage composition was calculated from the GC peak areas using the normalization method (without correction factors). The mixture of $\mathrm{C}_{5}-\mathrm{C}_{24} n$ alkanes was injected using the above mentioned temperature program in order to calculate the retention indices. ${ }^{20}$ Identification of the components was based on computer matching their mass spectral fragmentation patterns with those in NIST05 and Wiley (Flavor \& Fragrance Natural \&Synthetic Compounds 1.2) Library.

\subsection{Antioxidant activity determination in vitro}

In DPPH radical scavenging, ABTS radical scavenging and ferric reducing antioxidant power assaying experiments, Trolox in concentrations ranging from 50 to $800 \mu \mathrm{M} \mathrm{L}^{-1}$ was used to construct standard curve. The tested antioxidant powers were expressed as Trolox equivalent (TE) antioxidant capacity in mMTE $\mathrm{L}^{-1}$. All the antioxidant testing experiments were conducted in triplicate.

2.5.1. Scavenging DPPH radicals. The DPPH radical scavenging activity of the EO was determined as the way of Avanço et al. ${ }^{21}$ with minor modifications. Initially, $2850 \mu \mathrm{L}$ of a solution of the DPPH radical in methanol was added to $150 \mu \mathrm{L}$ of EO 
dilutions in methanol at needed concentrations. The reaction occurred for $8 \mathrm{~h}$ in the dark at room temperature, following which absorbance was read at $515 \mathrm{~nm}$ using a UV-VIS 2450 spectrophotometer (Shimadzu, Japan).

2.5.2. ABTS radical scavenging assay. The ABTS radical cation $\left(\mathrm{ABTS}^{\cdot+}\right)$ bleaching assay was carried out as reported by Carrasco et al. ${ }^{22}$ with slight modifications. $\mathrm{ABTS}^{{ }^{++}}$was produced by reacting equal volumes of $7 \mathrm{mM}$ of ABTS with $2.5 \mathrm{mM}$ potassium persulfate and leaving the mixture in the dark at room temperature for $15 \mathrm{~h}$ before use. The $\mathrm{ABTS}^{\cdot+}$ solution was diluted $(1: 100)(\mathrm{v} / \mathrm{v})$ with ethanol to $0.035 \mathrm{mM}$ with an absorbance of $0.7 \pm 0.02$ at $734 \mathrm{~nm}$. Methanolic solutions of the EOs were mixed $(1: 4)(\mathrm{v} / \mathrm{v})$ with the prepared $\mathrm{ABTS}^{{ }^{+}}$solution. After reaction at room temperature for $6 \mathrm{~min}$, the absorbance at $734 \mathrm{~nm}$ was measured via Spectrophotometer 1510 (Thermo Fisher scientific) using a 96-well plate. Lower absorbance of the reaction mixture indicates higher $\mathrm{ABTS}^{*+}$ scavenging activity.

2.5.3. Ferric reducing antioxidant power(FRAP). The FRAP assay was conducted according to the way of Aissi et al. ${ }^{23}$ with mere modifications. The fresh FRAP reagent was prepared by mixing TPTZ solution (10 $\mathrm{mM}$ TPTZ in $40 \mathrm{mM} \mathrm{HCl}$ ), ferric solution $\left(20 \mathrm{mM}, \mathrm{FeCl}_{3} \cdot 6 \mathrm{H}_{2} \mathrm{O}\right)$, and acetate buffer $(300 \mathrm{mM}, \mathrm{pH}$ 3.6) in the proportions $1: 1: 10(\mathrm{v} / \mathrm{v} / \mathrm{v})$. The reaction was then performed by mixing $2.8 \mathrm{~mL}$ of FRAP solution with $0.2 \mathrm{~mL}$ of the methanol diluted EO samples. The mixture was incubated at $37{ }^{\circ} \mathrm{C}$ for $30 \mathrm{~min}$. The absorbance at $593 \mathrm{~nm}$ was determined against methanol as a blank.

2.5.4. $\quad \beta$-Carotene bleaching test (BCBT). As described by Jallali et al. ${ }^{24} \beta$-carotene $(2 \mathrm{mg}$ ) was dissolved in $20 \mathrm{~mL}$ chloroform. An aliquot of this solution ( $4 \mathrm{~mL}$ ) was mixed with $40 \mathrm{mg}$ linoleic acid and $400 \mathrm{mg}$ Tween 40 . Chloroform was evaporated under vacuum at $40^{\circ} \mathrm{C}$ and $100 \mathrm{~mL}$ of oxygenated distilled water was added and then ultrasonically emulsified. An aliquot (150 $\mu \mathrm{L}$ ) of the above emulsion was distributed in the 96-well plates and EO samples $(10 \mu \mathrm{L})$ were added. The plates were incubated at $50{ }^{\circ} \mathrm{C}$ for $120 \mathrm{~min}$, and the absorbance was measured via Spectrophotometer 1510 (Thermo Fisher scientific) at $470 \mathrm{~nm}$. Readings of all samples were performed immediately ( $0 \mathrm{~min}$ ) and after $120 \mathrm{~min}$ of incubation. The antioxidant activity (AA) of the samples was evaluated in term of $\beta$-carotene blanching using the following formula:

$$
\mathrm{AA}(\%)=\left[\left(A_{0}-A_{1}\right) / A_{0}\right] \times 100
$$

where $A_{0}$ is the absorbance of the control at $120 \mathrm{~min}$, and $A_{1}$ is the absorbance of the sample at $120 \mathrm{~min}$.

\subsection{Antifungal assays in vitro}

Four postharvest pathogenic fungi were used in the bioassay. Among them, Alternaria alternata was separated from naturally infected pear fruit marked black spot; Penicillium expansum was isolated from naturally blue mold occurred apples; Trichothecium roseum was isolated from decayed muskmelon fruit typically with pink mold; Fusarium sulphureum Schlechlendah, causal fungus of dry rot of potato tuber, was provided by the Institute of Plant Protection, Gansu Academy of Agricultural Sciences. All the fungal species were maintained on potato dextrose agar (PDA) plate at $4{ }^{\circ} \mathrm{C}$ and conidia of the pathogens were obtained as described by Deng et $a .^{25}$

2.6.1. Spore germination test. The modified method of Chitarra et al. ${ }^{26}$ was used to evaluate the effect of EOs on fungal spore germination. Different concentrations (0, 400, 800, 1200 and $1600 \mu \mathrm{L} \mathrm{L}^{-1}$ ) of EO amended potato dextrose broth (PDB) were prepared by dissolving the requisite amounts of sterile EOs $(0.2 \mu \mathrm{m}$ filter) into PDB containing $0.05 \%$ (v/v) Tween 80 and 0 concentrate one was used as control. Slides inoculated with 20 $\mu \mathrm{L}$ conidial suspension $\left(10^{6}\right.$ conidia per $\left.\mathrm{mL}\right)$ in the above PDB were placed on moist filter paper in Petri plates, sealed with Parafilm to avoid evaporation and incubated at $27^{\circ} \mathrm{C}$. When the germination percentage of the control suspensions of a fungus rose to 80 , all the corresponding treated slides of the fungus were immediately fixed with lactophenol cotton blue to stop further germinating and the germinated conidia of each slide were counted. The results were expressed as percent of spore germination inhibition using the formula:

$$
\mathrm{GI}(\%)=\left[\left(G_{\mathrm{c}}-G_{\mathrm{t}}\right) / G_{\mathrm{c}}\right] \times 100
$$

where $G_{\mathrm{c}}$ and $G_{\mathrm{t}}$ represent the mean number of germinated spores in control and treated slides, respectively. Each treatment was performed in triplicate.

2.6.2. Antifungal assay on mycelia growth. In vitro antifungal assays were carried out with a poisoned food technique ${ }^{27}$ with slight modifications. In short, EO were amended to sterile molten $\left(45-50{ }^{\circ} \mathrm{C}\right)$ PDA containing $0.1 \%(\mathrm{v} / \mathrm{v})$ Tween 80 to final concentrations of 0.5 and $1 \mu \mathrm{L} \mathrm{mL}^{-1}(\mathrm{v} / \mathrm{v})$ and poured into Petri plates (90 mm-diameter). Mycelia disks (6 mm-diameter) from the periphery of 7 day-old cultures were centrally inoculated on to the agar plates with the disks with mycelium surface facing down. The plates were parafilmed and then incubated at $27{ }^{\circ} \mathrm{C}$ in the dark for seven days. Control consisted of unamended PDA medium with $0.1 \%(\mathrm{v} / \mathrm{v})$ Tween 80 . The mean of two perpendicular diameters of the colony was measured. The percentage of mycelial radial growth inhibition was calculated with formula:

$$
\operatorname{MGI}(\%)=[(C-T) / C] \times 100
$$

where $C$ and $T$ represent mycelial growth diameter in control and EO-amended Petri plates, respectively. Three plates were used for each treatment as replications.

2.6.3. Determination of minimum inhibitory and fungicidal concentrations. The minimal inhibitory concentrations (MICs) of the EOs were determined by the agar dilution method essentially described by Boubaker et al. ${ }^{28}$ Briefly, molten PDAs with EO concentration from 4000 to $250 \mu \mathrm{L} \mathrm{L}^{-1}$ were dispensed into the Petri plates and solidify. Aliquots $(50 \mu \mathrm{L})$ of a spore suspension $\left(10^{6}\right.$ conidia per $\left.\mathrm{mL}\right)$ of each fungus were evenly inoculated on the PDA surface. The plates were parafilmed and incubated at $27{ }^{\circ} \mathrm{C}$ in dark for seven days. The lowest EO concentrations that allowed no visible growth of the pathogen were recorded as corresponding MICs.

The minimum fungicidal concentrations (MFCs) were determined by taking agar plugs from above MIC experiment 
plates showing no visible mycelial growth and reinoculating them upside down on unamended PDA medium. MFC was regarded as the lowest EO concentrations preventing growth of the pathogens after seven-day incubation. There were three replicates for each EO test at each concentration.

\subsection{Statistical analysis}

All the experimental data were expressed in terms of mean values and subjected to statistical analysis with Minitab v.17.1. Percentage values of inhibition of spore germination were subjected to arcsine square root transformation before analysis of variance. Mean separation was performed by the Duncan's multiple range test at $P<0.05$. All the graphs were drawn using the Origin Pro9 (OriginLab, MA, USA).

\section{Results and discussion}

\subsection{Remaining significant factors}

Among the variables tested, $\mathrm{HCl}$ concentration, sieving mesh and acidolysis duration had significant effects $(P<0.05)$ on EO yield (Table 4$)$. The coefficient of determination $\left(R^{2}=0.9822\right)$ indicates that $98.22 \%$ of the variability of the response could be explained by the model, so the goodness of fitting of the model was confirmed. The EO-yield-predicting polynomial model obtained from PBD regression analysis is expressed in terms of coded factors as:

$$
\begin{aligned}
& Y=2.5331-0.0826 X_{1}+0.0175 X_{2}+0.0427 X_{3}-0.0207 X_{4} \\
& -0.0180 X_{5}-0.0576 X_{6}+0.0827 X_{7}+0.1353 X_{8}+0.0736 X_{9}
\end{aligned}
$$

The Pareto chart (Fig. 1) illustrates that three parameters (acidolysis duration $\left(X_{8}\right), \mathrm{HCl}$ concentration $\left(X_{7}\right)$ and sieving mesh $\left(X_{1}\right)$ ) were significant factors. Microwave assistance applied in EO preparation in both solvent-free dry distillation $^{29,30}$ and simultaneous combination with hydrodistillation $^{11,31}$ can improve EO yield and shorten extraction time. Variations in microwave power and duration in the pretreatment of black cumin seeds can influence the oil

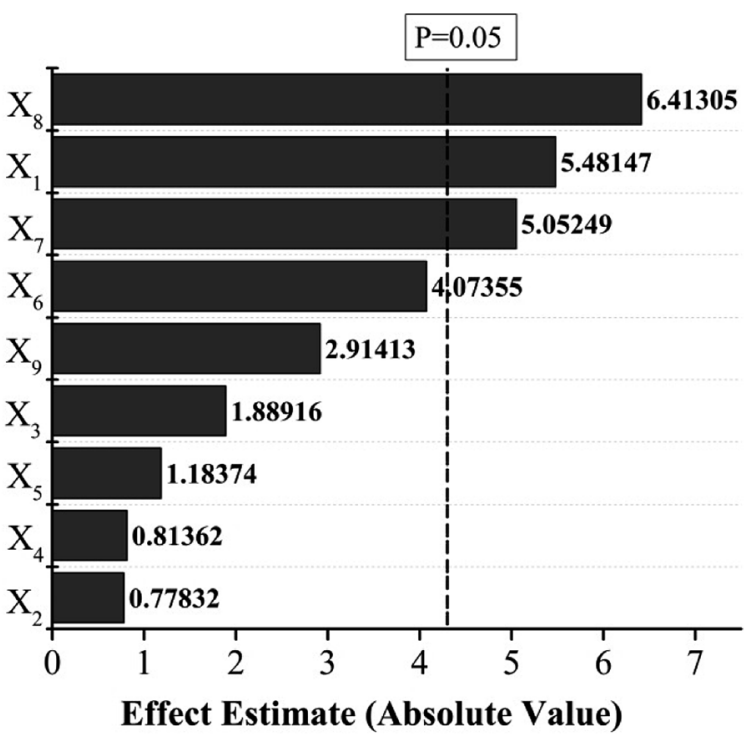

Fig. 1 Pareto chart to the independent variable effects on essential oil yield. $X_{1}=$ sieving mesh, $X_{2}=$ enzyme concentration $\left(10^{4} \cup \mathrm{L}^{-1}\right), X_{3}=$ enzymolysis $\mathrm{pH}, X_{4}=$ enzymolysis temperature $\left({ }^{\circ} \mathrm{C}\right), X_{5}=$ enzymolysis duration (min), $X_{6}=$ microwave irradiation duration $(\mathrm{s}), X_{7}=\mathrm{HCl}$ concentration of acidolysis $\left(\mathrm{ML}^{-1}\right), X_{8}=$ acidolysis duration (min), $X_{9}=$ sample to liquid ratio.

pressing efficiency from the seeds. ${ }^{32}$ In the present work, short time microwave pretreatment was statistically excluded from the significant factors determining the EO yield. Enzymatic pretreatment with cellulase alone or allied with hemicellulase can increase the EO yields of both Thymus capitatus and Rosmarinus officinalis and elevate content of the predominant component carvacol for $T$. capitatus but a drop in the content of main component 1,8 -cineole in the case of $R$. officinalis. ${ }^{33}$ In another case, spraying of the cumin seeds with cellulase solution increased its EO yield but changed the oil chemical profile. ${ }^{34}$ Back to the current exploration matrix, the influence of cellulase pretreatment upon EO yields was statistically regarded as insignificant.

\begin{tabular}{|c|c|c|c|c|c|}
\hline Term & $\mathrm{DF}$ & Adj. SS & Adj. MS & $F$-value & $P$-value \\
\hline Model & 9 & 0.156133 & 0.017346 & 20.48 & 0.047 \\
\hline Linear & 9 & 0.156113 & 0.017346 & 20.48 & 0.047 \\
\hline$X_{1}$ & 1 & 0.044380 & 0.044380 & 30.05 & 0.032 \\
\hline$X_{2}$ & 1 & 0.000895 & 0.000895 & 0.61 & 0.518 \\
\hline$X_{3}$ & 1 & 0.005238 & 0.005238 & 3.55 & 0.200 \\
\hline$X_{4}$ & 1 & 0.000978 & 0.000978 & 0.66 & 0.501 \\
\hline$X_{5}$ & 1 & 0.002070 & 0.002070 & 1.40 & 0.358 \\
\hline$X_{6}$ & 1 & 0.024510 & 0.024510 & 16.59 & 0.055 \\
\hline$X_{7}$ & 1 & 0.037705 & 0.037705 & 25.53 & 0.037 \\
\hline$X_{8}$ & 1 & 0.060747 & 0.060747 & 41.13 & 0.023 \\
\hline$X_{9}$ & 1 & 0.012543 & 0.012543 & 8.49 & 0.100 \\
\hline Error & 2 & 0.002954 & 0.001477 & & \\
\hline Total & 11 & 0.159067 & & & \\
\hline
\end{tabular}

Table 4 Analysis of variance (ANOVA) for the regression model of essential oil yield using the Plackett-Burman design

$S=0.0384323$

$R$-sq $=0.9822$

Adj. $R$-sq $=0.9023$ 
Table 5 Analysis of variances (ANOVA) for response surface quadratic model $^{a}$

\begin{tabular}{|c|c|c|c|c|c|}
\hline Source & $\mathrm{DF}$ & Adj. SS & Adj. MS & $F$-value & $P$-value \\
\hline Model & 9 & 1.586 & 0.176222 & 19.78 & $<0.0001$ \\
\hline Linear & 3 & 0.65858 & 0.219526 & 24.64 & $<0.0001$ \\
\hline$X_{1}$ & 1 & 0.02498 & 0.024976 & 2.8 & 0.138 \\
\hline$X_{2}$ & 1 & 0.37368 & 0.37368 & 41.94 & $<0.0001$ \\
\hline$X_{3}$ & 1 & 0.25992 & 0.25992 & 29.17 & 0.001 \\
\hline Square & 3 & 0.82068 & 0.273561 & 30.7 & $<0.0001$ \\
\hline$X_{1}^{2}$ & 1 & 0.58017 & 0.580166 & 65.11 & $<0.0001$ \\
\hline$X_{2}^{2}$ & 1 & 0.01087 & 0.010866 & 1.22 & 0.306 \\
\hline$X_{3}^{2}$ & 1 & 0.27175 & 0.271753 & 30.5 & 0.001 \\
\hline 2-way interaction & 3 & 0.010674 & 0.035581 & 3.99 & 0.06 \\
\hline$X_{12}$ & 1 & 0.01254 & 0.012544 & 1.41 & 0.0274 \\
\hline$X_{13}$ & 1 & 0.03294 & 0.032942 & 3.7 & 0.096 \\
\hline$X_{23}$ & 1 & 0.06126 & 0.061256 & 6.87 & 0.034 \\
\hline Error & 7 & 0.06237 & 0.00891 & & \\
\hline Lack-of-fit & 3 & 0.04723 & 0.015743 & 4.16 & 0.101 \\
\hline Pure error & 4 & 0.01514 & 0.003786 & & \\
\hline Total & 16 & 1.64837 & & & \\
\hline \multicolumn{6}{|l|}{$S=0.0943954$} \\
\hline \multicolumn{6}{|l|}{$R^{2}=0.9622$} \\
\hline \multicolumn{6}{|l|}{$R^{2}(\operatorname{Adj})=0.9135}$. \\
\hline
\end{tabular}

\subsection{BBD optimization of EO preparation}

3.2.1. Model fitting and analysis. The function law of the remaining parameters was determined from the analysis of variance and the goodness-of-fit of the model was tested by the coefficient of determination $\left(R^{2}\right)$ and adjusted coefficient of determination (Adj. $R^{2}$ ). The large $F$-value and concurrent $P<0.05$ (Table 5) indicate that the factor had a significant effect on the EO yield. As showed, the regression equation for EO yield was highly significant $(F=19.78, P<0.0001)$, demonstrating that the degree of fit was better on the border of the independent variables. The variable with the largest effect on EO yield was the quadratic term of mesh size $(F=65.11, P<0.0001)$, followed by the linear term of $\mathrm{HCl}$ concentration of acidolysis $(F=41.94, P<0.0001)$ and then the quadratic term of acidolysis duration $(F=30.5, P=0.001)$. The model $F$-value of $19.78(P<0.0001)$ also implies that the regression model was highly significant and confirmed that the model was congruent with the present experiment. A statistically significant confidence level of $99.999 \%, R^{2}(0.9622)$ and Adj. $R^{2}$ $(0.9135)$ prove the polynomial model was adequate to navigate the designed process of EO preparation.

The significance of each coefficient was determined by the $F$-value and the $P$-value. In this case, $X_{2}, X_{3}, X_{1}{ }^{2}, X_{3}{ }^{2}, X_{1} X_{2}$ and $X_{2} X_{3}$ were significant model terms $(P<0.05)$. The most effective variables were $\mathrm{HCl}$ concentration and acidolysis duration in the process. The coefficients of independent variables determined by the second-order polynomial model are given below (in terms of coded variables):

$$
\begin{gathered}
Y=2.9534+0.0559 X_{1}+0.2161 X_{2}-0.1803 X_{3}-0.3712 X_{1}^{2} \\
+0.0508 X_{2}^{2}+0.2541 X_{3}^{2}-0.0560 X_{1} X_{2}+0.0907 X_{1} X_{3} \\
-0.1238 X_{2} X_{3}
\end{gathered}
$$
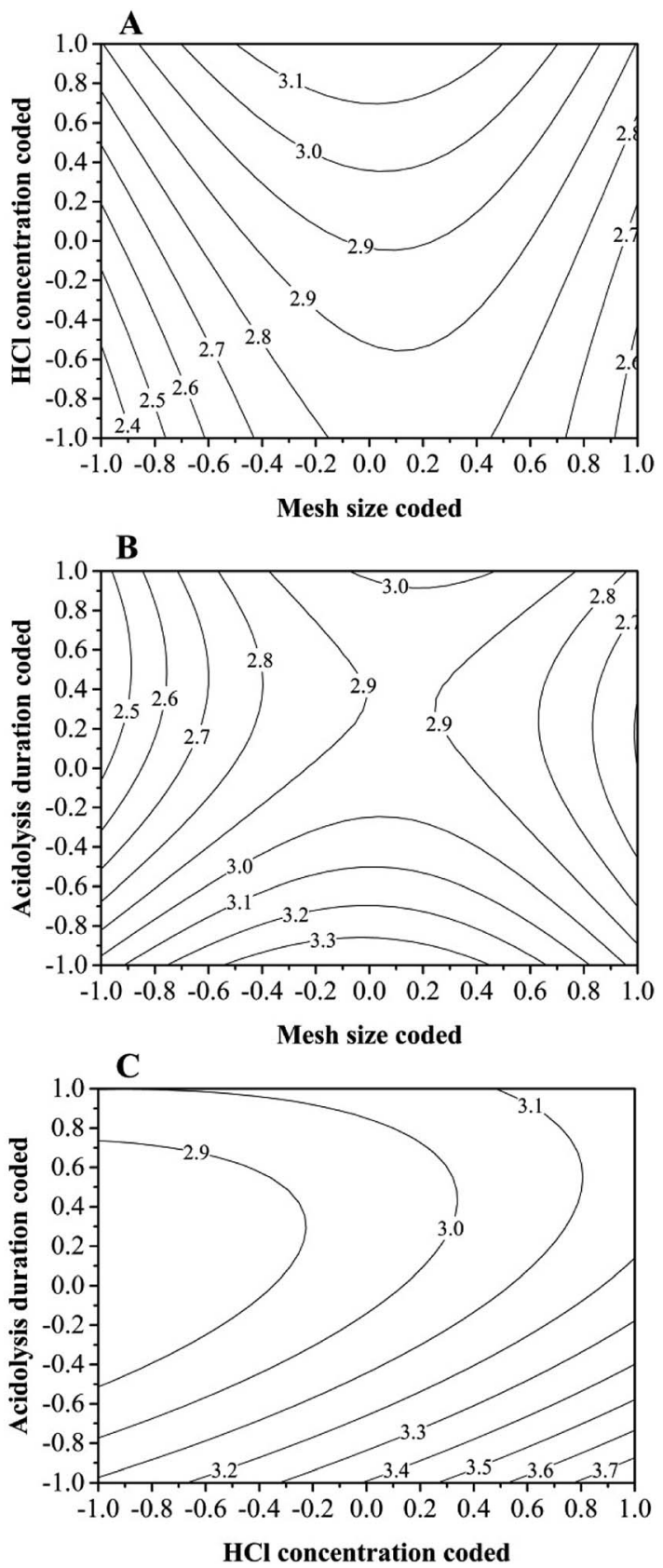

Fig. 2 Contour plots showing the interaction effects of treatment parameters and the influence on the $C$. cyminum seeds essential oil yield as predicted by BBD based response surface quadratic model with the acidolysis duration held constant at medium (60 min) value (A), $\mathrm{HCl}$ concentration held constant at medium $\left(2 \mathrm{ML}^{-1}\right)$ value (B), and mesh size held constant at medium (40 mesh) value (C)

where $X_{1}$ is cumin powder mesh size; $X_{2}$ is $\mathrm{HCl}$ concentration; $X_{3}$ is acidolysis duration.

3.2.2. Response contour analyses. The relationship and interaction between two independent variables and dependent variable could also be illustrated with contour plots. Fig. 2A-C demonstrate the impact of the remained treatment parameters on the EO yield, with the third parameter for each plot held at its medium value (60 $\mathrm{min}, 2 \mathrm{M} \mathrm{L}^{-1}$ or $40 \mathrm{mesh}$ ). 
The influence of $\mathrm{HCl}$ on the EO yield at a given mesh size is likely to increase as the concentration is increased (Fig. 2A). But too high $\mathrm{HCl}$ concentration is impractical because of the detrimental effects on the EO quality and operator. Mesh size had the best performance at its near medium value. This is possibly due to the two opposite effects of grinding process. On one hand, grinding increases the specific surface area of the sample and elevates the efficiency of acidolysis and EO releasing; on the other hand, over grinding makes the sample temperature high and evaporation causes yield losses.

Effects of acidolysis duration and mesh size on EO yield at a fixed acid concentration of $2 \mathrm{M} \mathrm{L}^{-1}$ were shown in Fig. 2B. Acidolysis duration and mesh size appear to have a more complex and saddle-like pattern of effect on the oil yield, while the interaction between them was positively correlated with yield. The figure displays that for any acidolysis durations, the EO yields reached the maximal response when the mesh size was at medium value. By moving along the $X$ and $Y$ axes, it can be observed that at the coded acidolysis duration of 0.4 and at the medium mesh size there is a saddle point where the EO yield response was low. Below the saddle-point acidolysis duration value, the lower the acidolysis duration was, the higher the EO yield might be.

Fig. 2C displays the relationship and interaction between the acidolysis duration and $\mathrm{HCl}$ concentration in the preparation of EO. Contour lines indicate that the influence of acidolysis duration could diminish after some critical values, suggesting that extended acidolysis duration more than necessary means loss of EO yield. It should be emphasized that the acid concentration and acidolysis duration had a positive quadratic effect on the oil yield.

3.2.3. Validation of the model and confirmatory test. The congruity of BBD based response surface quadratic model predictions and experimental values for the EO yield is revealed by the regression equation $Y=X+0.00001$ with $R^{2}=0.9622$, where $Y$ is the predicted yield and $X$ is the experimental yield. The coefficient of determination $\left(R^{2}\right)$ of the equation 0.9622 indicates that the model adequately fit the real relationships between the variables.

Theoretically it can be concluded from the regression equation that there is a combination zone of certain values of mesh size, $\mathrm{HCl}$ concentration and acidolysis duration where a highest EO yield response could be achieved. With the analyses of gained response contours and considering the damaging effects of too high $\mathrm{HCl}$ concentration on the EO quality, a compromise concentration was reached to be $2.5 \mathrm{M}$ $\mathrm{L}^{-1}$. The optimized mesh size and acidolysis duration were 40 mesh and $45 \mathrm{~min}$, respectively. Under above extraction conditions, the model response was $3.78 \%$. This prediction was well validated by the value $3.86 \%$ achieved by confirmatory experiments under above optimized conditions.

Certain plant volatile compounds exist as glycosidically bound components ${ }^{\mathbf{1 4}}$ and most of these glycosidic compounds contain aglycone moieties belonging to different classes of metabolites with a preponderance of phenols and terpenoids. ${ }^{35}$ Glycosylation of lipophilic volatile compounds keeps the labile cellular components from damage and protects plants themselves from toxicity. ${ }^{36}$ After being bound, these otherwise hydrophobic substances could achieve a good accumulation, transport, and storage. ${ }^{37}$ Subjected to acidolysis or enzymolysis, the glycosidic precursors can yield free volatiles. ${ }^{38}$ Seldom does conventional distillation without proper hydrolysis consider the liberating of bound volatiles in obtaining of EO. In the current work, acidolysis is assumed to break the glycosidical bound and assist the releasing of hydrophobic aglycones that can then be easily included in the EOs.

\subsection{Impact on EO yield and composition}

3.3.1. Elevation of EO yield. After the PBD and BBD based process optimization, acidolysis-pretreated cumin gained an EO yield of $3.86 \%$ while the control sample only gained $2.56 \%$. This result indicates that the optimized pretreatments had a highly significant effect on the EO yield $(P<0.01)$, which conferred the treated sample an increase of $50.78 \%$ in EO yield over control sample.

3.3.2. Alteration of EO chemical profile. Totally 53 components were identified in the acidolysis-pretreated cumin EO (AEO) whilst 47 components were in control cumin EO (CEO) with relative peak area $>0.01 \%$ (Table 6). In both EO samples, cuminaldehyde was the predominant component in common with a percentage of 25.14 and 21.89 for the AEO and CEO, respectively, but a distinction existed between the chemical profiles of them as tangibly indicated by their GC-MS chromatograms (Fig. 3A and B). By comparison, cymene, $\gamma$-terpinene and cuminaldehyde are the first three major components of Indian cumin seeds EO. ${ }^{4}$ The major constituents of one Iranian commercial cumin EO are $p$-cymene, $\gamma$-terpinene, $\beta$-pinene and cuminaldehyde. ${ }^{39}$ Earlier report $^{40}$ showed that cuminaldehyde, cuminic alcohol and $\gamma$ terpinene are three most predominant components of EO of cumin sample from Urumuqi, China. This discrepancy was in agreement with the statement ${ }^{41}$ that certain variability exists in chemical composition of spice EOs due to several factors including then climate, sampling season, growth location, exact botanical parts and extraction ways. In the current research, a total of 24 compounds with relative high abundance were shared by AEO and CEO. Meanwhile some mere compounds were only detected in individual sample. From a view of the compound types, alkenes (47.07\%) predominated the CEO followed by aldehydes (41.49\%), and this situation was opposite in AEO with aldehydes (45.72\%) and alkenes $(34.68 \%)$. Another two types of compounds, although not abundant, phenols and alcohols presented more in AEO than in CEO (Table 6), which was probably resulted from the hydrolysis of their bound precursors. It is reported that certain lipophilic volatile compounds mainly phenols and alcohols are damaging to cell and glycosylation of them can diminish their toxicity. ${ }^{36}$

\subsection{Antioxidant activity}

As demonstrated (Table 7), the DPPH and ABTS radical scavenging activities of AEO were 2.41 and 4.11-fold of that of CEO, 
Table 6 Chemical composition of acidolysis-pretreated cumin EO (AEO) and control cumin EO (CEO) ${ }^{a}$

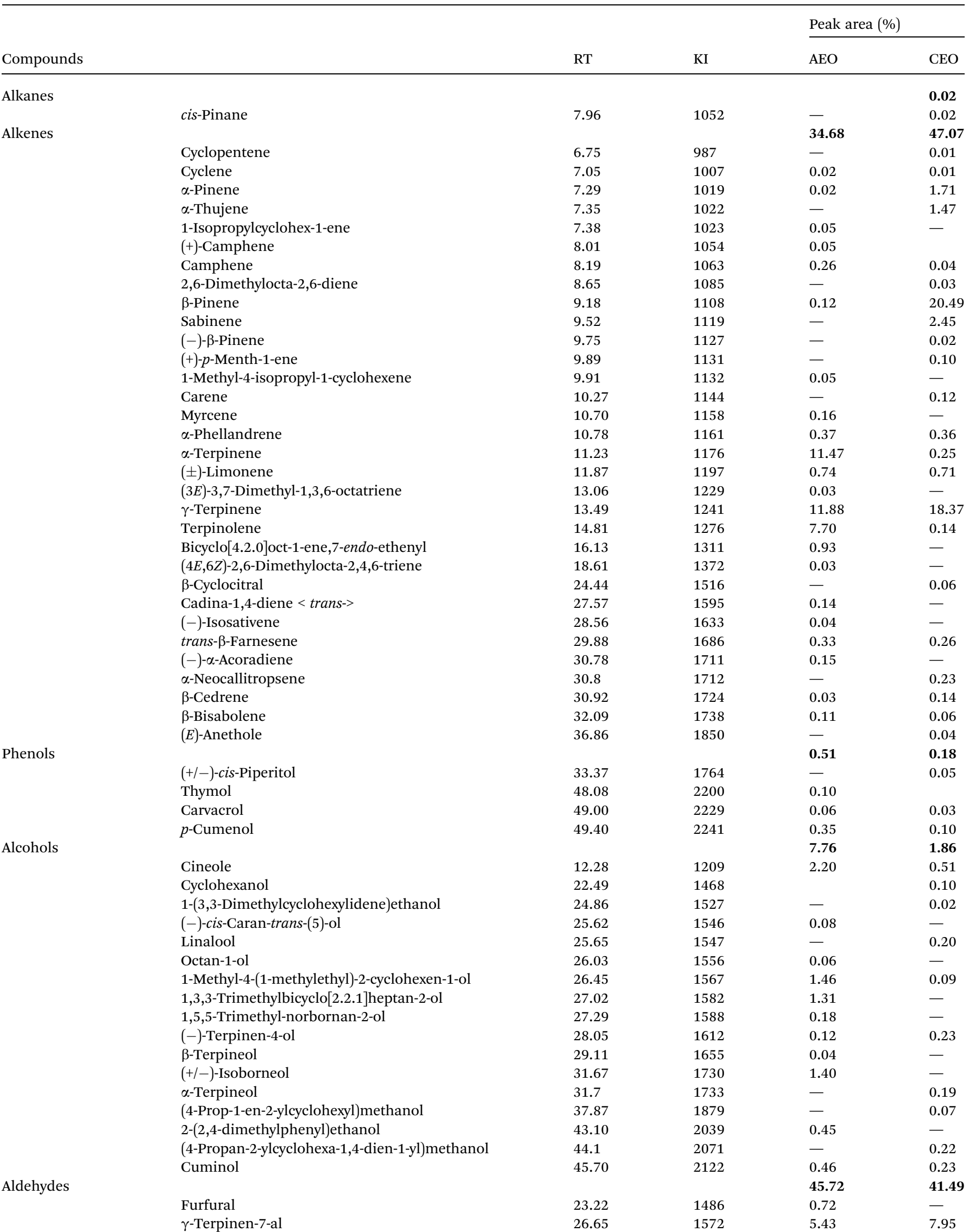


Table 6 (Contd.)

\begin{tabular}{|c|c|c|c|c|c|}
\hline \multirow[b]{2}{*}{ Compounds } & & \multirow[b]{2}{*}{$\mathrm{RT}$} & \multirow[b]{2}{*}{ KI } & \multicolumn{2}{|c|}{ Peak area $(\%)$} \\
\hline & & & & $\mathrm{AEO}$ & CEO \\
\hline & Phellandral & 32.88 & 1754 & 1.50 & 0.64 \\
\hline & Cuminaldehyde & 35.08 & 1799 & 25.14 & 21.89 \\
\hline & $\gamma$-Terpinen-7-al & 35.57 & 1813 & 12.74 & 10.98 \\
\hline & Terpinylformate & 24.37 & 1514 & 0.06 & - \\
\hline & trans-Pinocarveyl acetate & 29.63 & 1676 & - & 0.03 \\
\hline & $d$-Sabinyl-acetate[trans-] & 48.87 & 2225 & 0.07 & - \\
\hline \multirow[t]{3}{*}{ Ketones } & & & & 0.59 & 0.00 \\
\hline & trans-4-Caranone & 28.13 & 1615 & 0.03 & - \\
\hline & (-)-cis-Carane-4-one & 28.72 & 1639 & 0.17 & - \\
\hline & Ocimenequintoxide & 12.65 & 1218 & 0.05 & - \\
\hline & p-Cymene & 14.49 & 1268 & 8.99 & 8.89 \\
\hline & 2-p-Tolyl-1-propene & 21.43 & 1442 & 1.22 & - \\
\hline & Daucene & 23.03 & 1481 & 0.33 & 0.23 \\
\hline & $(-)$-Germacrene-D & 24.93 & 1529 & - & 0.04 \\
\hline & Ionene & 29.66 & 1677 & 0.04 & - \\
\hline & Isodaucene & 32.29 & 1742 & - & 0.05 \\
\hline
\end{tabular}

respectively. The ferric reducing antioxidant power of AEO was 73.75 times as strong as that of CEO. In $\beta$-carotene bleaching, the AEO achieved a value 3.20 times as much as CEO did. The four antioxidant testing methods employed in the current work evaluated the sample antioxidant capacities based on different chemical mechanisms. The AEO displayed highly significantly stronger antioxidant capacities in every assay conducted than the CEO $(P<0.01)$. This might be due to the relative higher content of phenols in AEO, and this type of compound has particular antioxidant properties to delay or stop the aerobic oxidation of organic matter. It has been generally recognized that phenolic compounds act as antioxidants due to their high reactivity with peroxyl radicals, which are quenched by formal hydrogen atom transfer. ${ }^{42}$ In contrast, the CEO included more non-phenolic terpenoids that can easily undergo autoxidation to form unsaturated lipids. ${ }^{43}$ Thus poor antioxidant protection can be expected from CEO because products arising from autoxidation of these components or their cooxidation with substances to be protected in the matrices are reactive species with potential of propagating the oxidative chain. ${ }^{44}$ When obtained from the same botanical material but with different extraction techniques, the EOs with more phenolic compounds might hold stronger antioxidant potency than rivals containing more non-phenolic terpenoids, particularly unsaturated ones. ${ }^{45,46}$

\subsection{Antifungal activity analysis}

3.5.1. Effect on spore germination. The inhibitive activities of two EO samples against spore germination of tested fungi demonstrated a dose-dependent tendency (Table 8).

As to this spore static effect of both EOs at a particular concentration, a rough rule is that $T$. roseum, F. sulphureum and P. expansum are more sensitive to cumin EO than A. alternata. For each tested strain, there was at least one treatment concentration at which the two EOs showed significant difference in spore static efficacy. Particularly, this efficacy presented highly significant difference $(P<0.01)$ between the two EOs when assayed against $T$. roseum $\left(400 \mu \mathrm{L} \mathrm{L}^{-1}\right), F$. sulphureum $\left(1600 \mu \mathrm{L} \mathrm{L}^{-1}\right)$ and P. expansum $\left(800 \mu \mathrm{L} \mathrm{L}^{-1}\right)$.

These differences in spore static capacities are connected with their compositional distinction. The relationship between the EO composition especially dominating components and its antimicrobial activities was well documented. Compounds, like phenols, possessing a system of delocalized electrons (aromatic ring) and a hydroxyl group display higher antimicrobial activities than other EO constituents. ${ }^{47}$ EOs containing more phenols or aldehydes manifested the highest antimicrobial activity followed by EOs containing terpene alcohols, whereas other EOs containing terpene hydrocarbons were generally ineffective. ${ }^{48-50}$ Among other constituents, the monoterpene phenols and aldehyde derivatives were found to be the most active constituents in the EOs, and other terpene alcohols and hydrocarbons 

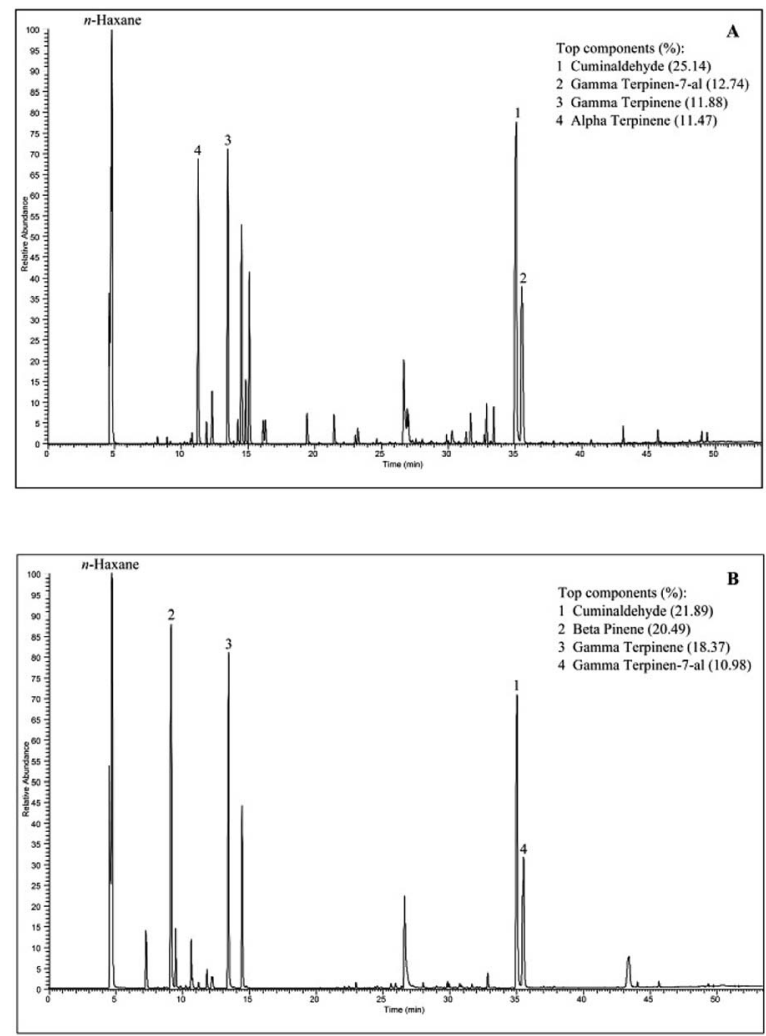

Fig. 3 GC-MS chromatogram comparison of C. cyminum essential oil $A E O(A)$ and $C E O(B)$. The top predominant components with relative percentage $>10$ of individual EO sample are listed in the respective frames.

like $\alpha$-pinene, 1 , 8-cineole, terpinene-4-ol and $\gamma$-terpinene were generally found to have moderate or weak antifungal activity. ${ }^{51}$

3.5.2. Inhibition efficacy on mycelia growth. On EO poisoned agars, all four tested fungi reduced their colony growth (Fig. 4). The inhibitive activities of the two EOs against every different fungus demonstrated significant differences at the concentration of $0.5 \mu \mathrm{L} \mathrm{mL} \mathrm{m}^{-1}(P<0.05)$. At this concentration, a maximal inhibition of $85.5 \%$ was observed on $F$. sulphureum treated with AEO and a minimal inhibition of only $10.4 \%$ was displayed on $P$. expansum poisoned with CEO. In contrast, the mycelial growth inhibition showed almost no difference and neared the observed maximum of $93 \%$ at $1.0 \mu \mathrm{L}$ $\mathrm{mL}^{-1}$. The AEO had stronger antifungal efficacy than the CEO against tested fungi at low concentration of $0.5 \mu \mathrm{L} \mathrm{mL}{ }^{-1}$.

Using a volatile phase method, Zamani-Zadeh and coworkers $^{39}$ evaluated the antifungal activity of cumin EO against pathogenic fungus Botrytis spp. and proved that the activity is dose-dependent but ineffective below the concentration of $3 \mu \mathrm{L} \mathrm{mL}{ }^{-1}$. With the food poison technique, Behdani et $a .^{52}$ determined the strong effect of cumin EO against $B$. cinerea mycelial growth on PDA culture even at the concentration of $0.25 \mu \mathrm{L} \mathrm{mL}^{-1}$.

3.5.3. MIC and MFC. The MICs of AEO were only one half, at most, of those of CEO for the tested fungi and the MFCs of the AEO were also lower than the counterpart MFCs of the CEO (Table 9).

Among the recorded MICs the least MIC $\left(1000 \mu \mathrm{L} \mathrm{L}^{-1}\right)$ was on the AEO against A. alternata, T. roseum and F. sulphureum, but this value against $P$. expansum was $2000 \mu \mathrm{L} \mathrm{L} \mathrm{L}^{-1}$. By comparison, the least MIC of $2000 \mu \mathrm{L} \mathrm{L}^{-1}$ was only measured on CEO against $T$. roseum and the value was two times folded when it came to other three fungi. The best fungicidal effect was demonstrated by AEO against T. roseum with an MFC value of $2000 \mu \mathrm{L} \mathrm{L}^{-1}$ while this value was $4000 \mu \mathrm{L} \mathrm{L}^{-1}$ against the remaining three fungi. In contrast, the MFCs $\left(>4000 \mu \mathrm{L} \mathrm{L}^{-1}\right)$ of CEO against all four fungi were outperformed by that of AEO. Simply put, the AEO had stronger antifungal activities than the CEO against four postharvest fungi tested. Interestingly, after transferring the inhibited Botrytis spp. mycelia disk from PDA treated with vapor phase cumin EO at MIC to non-treated PDA, Zamani-Zadeh et al. ${ }^{39}$ observed no mycelial growth and thereby determined the fungicidal nature of the cumin EO against the fungus.

Presumably the variance in the chemical composition of EOs like 'AEO' and 'CEO' could account for the differences in their bioactivities, but determination of the exact responsible components seems not easy. When we emphasized the key roles of certain compounds that can individually demonstrate noticeable activities, the holistic effect of different components of the EO should not be neglected. Milos and Makota ${ }^{53}$ investigated the antioxidant synergisms and antagonisms among thymol, carvacrol, thymoquinone and $p$-cymene, and concluded that majority of mixtures displayed some discrepancy in antioxidant capacity when compared to that of their individual constituents. Multiple components of EO partake in a bioactivity performance but they may have different function targets or mechanisms, and in some cases even exist offsetting between positive and negative effects. Nonetheless, the compositional information of EOs could adduce evidence in the rough comparison of some of their bioactivities. The relative antifungal activities of EOs might be tentatively judged according to their predominant components content as following: phenols > alcohols $>$ aldehydes $>$ ketones $>$ ethers $>$ hydrocarbons ${ }^{54}$ All these views were well mirrored in the relationship of compositional profile and bioactivities of the AEO and CEO, two preparations from the same botanical material.

The seeking a higher yield of EO extraction never stops since our ancestors' invention of the earliest EO distillation process.

Table 7 Antioxidant activity of acidolysis-pretreated cumin EO (AEO) and control cumin EO (CEO) evaluated with four methods ${ }^{a}$

\begin{tabular}{|c|c|c|c|c|}
\hline EOs & $\mathrm{DPPH}\left(\mathrm{mMTE} \mathrm{L}^{-1}\right)$ & $\operatorname{ABTS}\left(\mathrm{mMTE} \mathrm{L} \mathrm{L}^{-1}\right)$ & $\operatorname{FRAP}\left(\mathrm{mMTE} \mathrm{L}^{-1}\right)$ & ВСВТ(\%) \\
\hline AEO & $92.84 \pm 11.93^{* *}$ & $231.34 \pm 2.88^{* *}$ & $159.31 \pm 0.76^{* *}$ & $55.17 \pm 5.97^{* *}$ \\
\hline CEO & $38.49 \pm 11.92$ & $56.27 \pm 13.97$ & $2.16 \pm 1.03$ & $17.24 \pm 10.34$ \\
\hline
\end{tabular}

${ }^{a}$ TE: trolox equivalent; **: $P<0.01$ values are means \pm SD. 
Table 8 Antifungal effect of acidolysis-pretreated cumin EO (AEO) and control cumin EO (CEO) on spore germination of four postharvest fungi expressed as inhibition of spore germination (\%) ${ }^{a}$

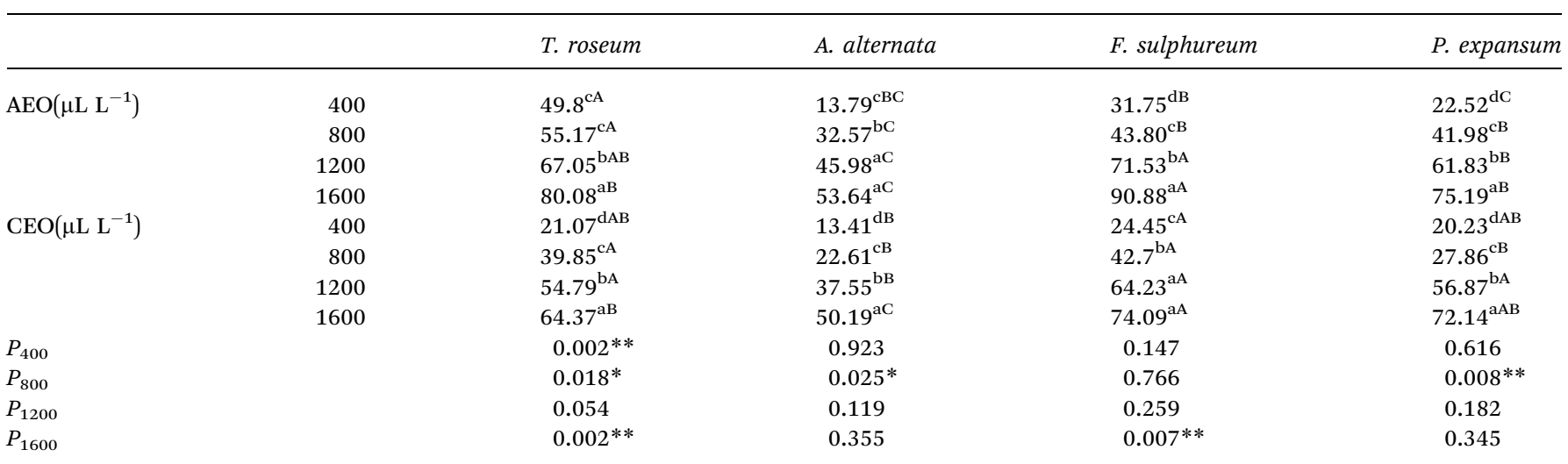

${ }^{a}$ Each value represents the mean of three replicates. Peer data in same row of a subgroup with differing superscript lower cases are significantly different $(P<0.05)$ between two concentrations of the same preparation. Peer data in same column with differing superscript capitals are significantly different $(P<0.05)$ between tested fungi at same EO concentration. $P$ values with shoulder "** and "*** indicating significant difference $(P<0.05)$ and highly significant difference $(P<0.01)$, respectively, between two preparations at same concentration.

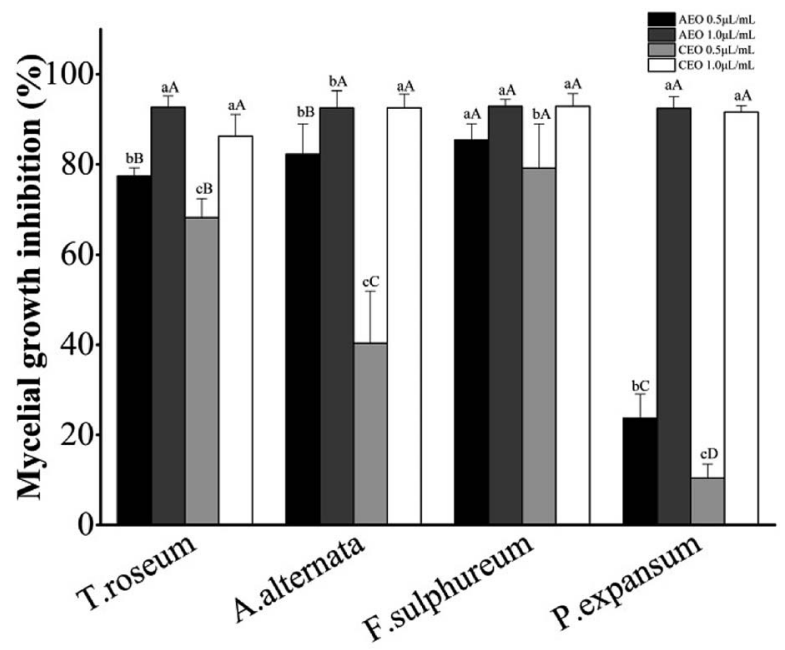

Fig. 4 In vitro effects of acidolysis-pretreated cumin EO (AEO) and control cumin EO (CEO) on mycelia growth of four postharvest fungi. Bioassay was conducted on essential oil-amended potato dextrose agar at 0.5 and $1.0 \mu \mathrm{L} \mathrm{mL}^{-1}$. Values are means \pm SD of three replicates. For an individual fungus, letters in lower case mean significant difference $(P<0.05)$ between different treatments considering the concentrations and EO preparations. For treatment with same concentration and preparation, different capitals indicate significant difference $(P<0.05)$ on tested fungus according to Duncan test.

Particularly in recent couple of decades, modern high technology and sophisticated equipment conferred researchers and industry more opportunities to elevate the EO yield to an even higher degree. But this dependence on fashionable technology and equipment often renders the extraction costly. Revisiting some traditional means may advance our understanding and application of these technical arsenals during the EO preparation. Acidolysis is an economical operation extensively utilized in biochemical industries especially for the pretreatment of raw materials, but until now, it was not reported in pretreatment of herbal materials for the EO distillation. To our knowledge, the present work is a fresh report on the application of acidolysis
Table 9 Antifungal activities expressed in MICs $\left(\mu L^{-1}\right)$ and MFCs $(\mu \mathrm{L}$ $\mathrm{L}^{-1}$ ) of acidolysis-pretreated cumin EO (AEO) and control cumin EO (CEO) to the bioassay fungi ${ }^{a}$

\begin{tabular}{llllll}
\hline EOs & & T. roseum & A. alternata & F. sulphureum & P. expansum \\
\hline \multirow{2}{*}{ AEO } & MIC & 1000 & 1000 & 1000 & 2000 \\
& MFC & 2000 & 4000 & 4000 & 4000 \\
CEO & MIC & 2000 & 4000 & 4000 & 4000 \\
& MFC & $>4000$ & $>4000$ & $>4000$ & $>4000$
\end{tabular}

${ }^{a}$ MIC: minimum inhibitory concentration; MFC: minimum fungicidal concentration.

pretreatment to the EO extraction and thereby the influences on the EO yield, composition and bioactivities.

The current investigation intended to integrate acidolysis, pulverization, enzymolysis and microwave irradiation into the pretreatment of cumin seeds prior to distillation. After statistical analysis based on PBD and BBD designs, two relative new arsenals, enzymolysis and short time microwave irradiation were screened out, and the two conventional technical elements, acidolysis and pulverization remained and their best performing zone were also determined. When properly applied, the two technical elements offered the following hydrodistillation a more than $50 \%$ increase of EO yield, this elevation were brilliant in comparison to that made by most of other technical improvements documented. Due to the liberation of more otherwise bound phytochemicals, remarkable species of new components were procured in the EO, which imparted the EO impressive variation of chemical profile, and enhanced antioxidant and antifungal bioactivities.

\section{Conclusion}

Acidolysis and pulverization extent have significant effects on the EO yield of cumin seeds. The optimal pretreatment was: 
pulverizing the seeds to 40 mesh and conducting $45 \mathrm{~min}$ acidolysis in $2.5 \mathrm{M} \mathrm{L}^{-1} \mathrm{HCl}$, wherein the predicted EO yield $3.78 \%$ was near close with the experimental value $3.86 \%$. The acidolysis-dominated pretreatment gave the seeds an EO-yield increase of $50.78 \%$ over the control sample (2.56\%). AEO and CEO shared the predominant component cuminaldehyde, but they had different chemical profiles. The AEO showed significantly higher antioxidant capability in DPPH radical scavenging, ABTS radical scavenging and ferric reducing antioxidant power assays and $\beta$-carotene bleaching test than CEO did. Additionally, the antifungal activities of AEO against four postharvest pathogenic fungi, viz. A. alternata, P. expansum, T. roseum and F. sulphureum outperformed that of CEO in most circumstances. This surpassing of bioactivities of AEO is resulted from its chemical characteristics imparted by the acidolysis-dominated pretreatment. The application of this novel pretreatment might be trialed on other aromatic plant materials to achieve valorization of their EO exploitation.

\section{Conflicts of interest}

There are no conflicts of interest to declare.

\section{Acknowledgements}

This work is financed by National Natural Science Foundation of China (31360416), Key Sci-tech Problem Tackling Project on Chinese Materia Media Industry of Gansu Province (2014) and Agricultural Sci-tech Innovation Program (GNCX-2012-42) of Gansu Province.

\section{References}

1 H. Hajlaoui, H. Mighri, E. Noumi, M. Snoussi, N. Trabelsi, R. Ksouri and A. Bakhrouf, Food Chem. Toxicol., 2010, 48, 2186-2192.

2 S. Mnif and S. Aifa, Chem. Biodiversity, 2015, 12, 733-742.

3 T. Allahghadri, I. Rasooli, P. Owlia, M. J. Nadooshan, T. Ghazanfari, M. Taghizadeh and S. D. A. Astaneh, J. Food Sci., 2010, 75, H54-H61.

4 A. Kedia, B. Prakash, P. K. Mishra and N. K. Dubey, Int. J. Food Microbiol., 2014, 168-169, 1-7.

5 R. M. O. F. Sousa, J. S. Rosa, L. Oliveira, A. Cunha and M. Fernandes-Ferreira, Ind. Crops Prod., 2015, 63, 226-237.

6 J. Wei, X. Zhang, Y. Bi, R. Miao, Z. Zhang and H. Su, J. Evidence-Based Complementary Altern. Med., 2015, DOI: 10.1155/2015/474509.

7 A. Tsoupras, R. Lordan and I. Zabetakis, Nutrients, 2018, 10, DOI: 10.3390/nu10050604.

8 U. E. Bauer, P. A. Briss, R. A. Goodman and B. A. Bowman, Lancet, 2014, 384, 45-52.

9 R. Ascrizzi, J. González-Rivera, C. S. Pomelli, C. Chiappe, P. Margari, F. Costagli, I. Longo, M. R. Tiné, G. Flamini and C. Duce, React. Chem. Eng., 2017, 2, 577-589.

10 T. Fornari, G. Vicente, E. Vázquez, M. R. García-Risco and G. Reglero, J. Chromatogr. A, 2012, 1250, 34-48.
11 M. M. A. Rashed, Q. Tong, A. Nagi, J. P. Li, N. U. Khan, L. Chen, A. Rotail and A. M. Bakry, Ind. Crops Prod., 2017, 100, 236-245.

12 L. K. Sharma, D. Agarwal, S. S. Rathore, S. K. Malhotra and S. N. Saxena, J. Food Sci. Technol., 2016, 53, 2827-2834.

13 R. Kowalski, G. Kowalska, J. Jamroz, A. Nawrocka and D. Metyk, Ultrason. Sonochem., 2015, 24, 214-220.

14 B. Sgorbini, C. Cagliero, A. Pagani, M. Sganzerla, L. Boggia, C. Bicchi and P. Rubiolo, Phytochemistry, 2015, 117, 296-305.

15 Y. Zhai, S. Sun, Z. Wang, J. Cheng, Y. Sun, L. Wang, Y. Zhang, H. Zhang and A. Yu, J. Sep. Sci., 2009, 32, 3544-3549.

16 S. Behera, S. Nagarajan and L. Jagan Mohan Rao, Food Chem, 2004, 87, 25-29.

17 H. Xiang, L. Zhang, L. Xi, Y. Yang, X. Wang, D. Lei, X. Zheng and X. Liu, Ind. Crops Prod., 2018, 111, 298-305.

18 Pharmacopoeia Committee of People's Republic China, Pharmacopoeia of People's Republic China, 2015.

19 T. Belwal, I. D. Bhatt, R. S. Rawal and V. Pande, Ind. Crops Prod., 2017, 95, 393-403.

20 R. Rodríguez-Solana, J. M. Salgado, J. M. Domínguez and S. Cortés-Diéguez, Ind. Crops Prod., 2014, 60, 186-192.

21 G. B. Avanço, F. D. Ferreira, N. S. Bomfim, P. A. de S. R. dos Santos, R. M. Peralta, T. Brugnari, C. A. Mallmann, B. A. de Abreu Filho, J. M. G. Mikcha and M. Machinski Jr, Food Control, 2016, 73, 1-8.

22 A. Carrasco, V. Ortiz-Ruiz, R. Martinez-Gutierrez, V. Tomas and J. Tudela, Ind. Crops Prod., 2015, 73, 16-27.

23 O. Aissi, M. Boussaid and C. Messaoud, Ind. Crops Prod., 2016, 91, 56-65.

24 I. Jallali, Y. Zaouali, I. Missaoui, A. Smeoui, C. Abdelly and R. Ksouri, Food Chem, 2014, 145, 1031-1038.

25 J. Deng, Y. Bi, Z. Zhang, D. Xie, Y. Ge, W. Li, J. Wang and Y. Wang, Postharvest Biol. Technol., 2015, 106, 53-61.

26 G. S. Chitarra, P. Breeuwer, M. J. R. Nout, A. C. Van Aelst, F. M. Rombouts and T. Abee, J. Appl. Microbiol., 2003, 94, 159-166.

27 A. Ben Ghnaya, I. Amri, M. Hanana, S. Gargouri, B. Jamoussi, A. Romane and L. Hamrouni, Ind. Crops Prod., 2016, 83, 113117.

28 H. Boubaker, H. Karim, A. El Hamdaoui, F. Msanda, D. Leach, I. Bombarda, P. Vanloot, A. Abbad, E. H. Boudyach and A. Ait Ben Aoumar, Ind. Crops Prod., 2016, 86, 95-101.

29 Q. Chen, Z. Hu, F. Y. D. Yao and H. Liang, LWT-Food Sci. Technol., 2016, 66, 538-545.

30 B. Bayramoglu, S. Sahin and G. Sumnu, J. Food Eng., 2008, 88, 535-540.

31 N. Jeyaratnam, A. H. Nour, R. Kanthasamy, A. H. Nour, A. R. Yuvaraj and J. O. Akindoyo, Ind. Crops Prod., 2016, 92, 57-66.

32 H. Bakhshabadi, H. Mirzaei, A. Ghodsvali, S. M. Jafari, A. M. Ziaiifar and V. Farzaneh, Ind. Crops Prod., 2017, 97, 1-9.

33 K. Hosni, I. Hassen, H. Chaâbane, M. Jemli, S. Dallali, H. Sebei and H. Casabianca, Ind. Crops Prod., 2013, 47, 291-299. 
34 H. B. Sowbhagya, P. Srinivas, K. T. Purnima and N. Krishnamurthy, Food Chem, 2011, 127, 1856-1861.

35 P. Winterhalter and G. K. Skouroumounis, Adv. Biochem. Eng./Biotechnol., 1997, 55, 73-105.

36 E. Stahl-Biskup, F. Intert, J. Holthuijzen, M. Stengele and G. Schulz, Flavour Fragrance J., 1993, 8, 61-80.

37 W. Hoesel, Biochem. Plants, 1981, 7, 725-753.

38 J. N. Ren, Y. N. Tai, M. Dong, J. H. Shao, S. Z. Yang, S. Y. Pan and G. Fan, Food Chem, 2015, 185, 25-32.

39 M. Zamani-Zadeh, S. Soleimanian-Zad, M. Sheikh-Zeinoddin and S. A. H. Goli, Postharvest Biol. Technol., 2014, 92, 149156.

40 R. Li and Z. T. Jiang, Flavour Fragrance J., 2004, 19, 311-313.

41 P. N. Dubey, S. N. Saxena, B. K. Mishra, R. K. Solanki, M. K. Vishal, B. Singh, L. K. Sharma, S. John, D. Agarwal and A. Yogi, Ind. Crops Prod., 2017, 95, 50-59.

42 M. C. Foti, J. Pharm. Pharmacol., 2007, 59, 1673-1685.

43 U. Neuenschwander, F. Guignard and I. Hermans, ChemSusChem, 2010, 3, 75-84.

44 R. Amorati, M. C. Foti and L. Valgimigli, J. Agric. Food Chem., 2013, 61, 10835-10847.
45 M. M. Esquível, M. A. Ribeiro and M. G. Bernardo-Gil, J. Supercrit. Fluids, 1999, 14, 129-138.

46 B. Marongiu, A. Piras, S. Porcedda, R. Casu and P. Pierucci, J. Essent. Oil Res., 2005, 17, 530-532.

47 A. Ultee, M. H. J. Bennik and R. Moezelaar, Appl. Environ. Microbiol., 2002, 68, 1561-1568.

48 A. Ait-Ouazzou, L. Cherrat, L. Espina, S. Lorán, C. Rota and R. Pagán, Innovative Food Sci. Emerging Technol., 2011, 12, 320-329.

49 J. Carneiro de Barros, M. Lúcia da Conceição, N. J. Gomes Neto, A. C. Vieira da Costa, J. P. Siqueira, I. D. Basílio and E. Leite de Souza, LWT-Food Sci. Technol., 2009, 42, 11391143.

50 M. M. Tajkarimi, S. A. Ibrahim and D. O. Cliver, Food Control, 2010, 21, 1199-1218.

51 M. Nikkhah, M. Hashemi, M. B. Habibi Najafi and R. Farhoosh, Int. J. Food Microbiol., 2017, 257, 285-294.

52 M. Behdani, M. Pooyan and S. Abbasi, Int. J. Agric. Crop Sci., 2012, 4, 1012-1016.

53 M. Milos and D. Makota, Food Chem, 2012, 131, 296-299.

54 P. Matusinsky, M. Zouhar, R. Pavela and P. Novy, Ind. Crops Prod., 2015, 67, 208-215. 